\title{
Note de l'éditeur
}

Les deux textes qui suivent sont les conférences données à Genève en novembre 2000 lors de la cérémonie en l'honneur du Prof. Pierre Montandon, qui quittait ses fonctions hospitalo-universitaires après 25 années de direction de la Clinique et Policlinique d'oto-rhino-laryngologie et de Chirurgie cervicofaciale des Hôpitaux universitaires de Genève.

Le Prof. Morgon montre bien que la pédo-audiologie est une discipline médicale à part entière. De plus en plus de praticiens équipent leur cabinet d'un ordinateur pour l'enregistrement et l'analyse automatique des potentiels évoqués auditifs et des oto-émissions acoustiques. Mais le diagnostic de surdité et le suivi des enfants réhabilités par une prothèse acoustique ou un implant cochléaire requièrent des compétences professionnelles spécifiques, que seule une équipe multidisciplinaire peut offrir. Le Prof. Pierre Montandon partageait cet avis. On lui doit en effet la création de l'unité de pédo-audiologie de la Clinique ORL des Hôpitaux universitaires de Genève.

Le Prof. Gacek décrit une affection rare, mais qu'il est important de connaître dans le diagnostic différentiel de l'otite séreuse chez l'adulte. Les contacts entre les membres de la Clinique ORL de Genève et le Prof. Gacek ont été étroits tout au long de ces décennies. Nous le remercions de l'enrichissement qu'il nous a apporté. Nous remercions aussi Pierre Montandon d'avoir su créer et maintenir des liens amicaux avec de nombreux otologistes internationaux de premier plan.

Jean-Philippe Guyot, Geneva

\begin{tabular}{ll}
\hline KARGER & @ 2003 S. Karger AG, Basel \\
1014-8221/03/0122-0088 \$19.50/0 \\
$\begin{array}{l}\text { Fax +4161306 12 34 } \\
\text { www. karger.com }\end{array}$ & $\begin{array}{l}\text { Accessible online at: } \\
\text { www.karger.com/orn }\end{array}$
\end{tabular}

